of low negative and positive density were found throughout the unit cell, hut the interesting fact remains that the highest peaks were found in the spaces between the heavy atoms. This is particularly the case for the $\mathrm{C}-\mathrm{C}$ bonds, while the terminal $\mathrm{C}-0$ bonds in the side chains have very small or absent residuals. To illustrate the situation the residual electron density in the planes of the rings is shown in Fig.8. Similar effects have been reported by Hartman \& Hirshfeld (1966) and have been more thoroughly discussed by OConnell. Rae \& Maslen (1966).

The writer is glad to have this opportunity of thanking Prof. O. Hassel and Prof. S. Furberg for the use of experimental equipment and for their kind interest in this work. I also owe C. Rømming and B. Klewe my gratitude for their advice and criticism, and A.Hordvik for encouraging discussions.

The financial support of Nansenfondet and of Norges Almenvitenskapelige Forskningstad is gratefully acknowledged.

\section{References}

Brow v, G. M. \& Levy, H. A. (1965). Science, 147, 1038. Buns, C. W. (1946). Chemical Crystallography, p.315. Oxford: Clarendon Press.

Cox. E. G. (1932). Nature, Lond. 130, 205.

Cox, F. G. \& Goobwin; T. H. (1936). J. Chem. Soc. p. 769.
Grison, E. (1951). Actn Cryst. 4, 489.

Hartman, A.\& Hirshifeld, F. L. (1966). Acta Cryst. 20, 80. Hamilton, W. C. (1955). Actn Cryst. 8, 185.

Haynes, L, J. \& Plimmer, J. R. (1960). Quart. Rel. Chem. Soc. Lond 14, 292.

Hendricks, S. B. (1934). Nature, Lond. 133, 178.

Herbert, R. W., Hirst, E. L., Percival, E. G. V., ReysOLDs, R.J. W. \& SMITH, F. (1933), ,.Chem. Soc. p. 1275.

HOLST, A. \& FRÖL ICH, T. (1907). Hy'g. (London), 7,619,634.

Hvoslef, J. (1964). Acta Chem. Scand. 18, 841

Jeffrey. C. A,. Rosenstein. R. D. \& Vlasse, M. (1967). Acta Cryst. 22, 725.

KiM, S. H., Jeffrey, G. A., Rosenstein, R. D. \& Cor* FIELD, P. W. R. (1967). Acta Cryst. 22, 733.

MacDonald, S. G. G. \& Alleyne, A. B. (1963). Acta Cryst. 16, 520 .

MCWefery, R. (1951). Acta Cryst. 4, 513.

Mathieson, A. MCL. \& TAYLOR, J. C. (1961). Tetrahedron Letters, 17, 592.

MATHIESON. A. MCL. (1963). Tetrahedron Letters, $2,81$.

NiGGI, E. (1942). Sichweitz. miner. petr. Miff. 22, 189.

O'Connell, A. M,, Rae, A. I. M. \& Maslen, E. N. (1966). Acta Cryst. 21, 208.

Steward, R, F., Davidoson, E. R. \& Simpson, N. T. (19651. J. Chem. Phys. 42, 3175.

StorchefF, B. P. (1962). Tetrahedron, 17, 135.

S7ent-Györgyi, A. (1928). Biochem, J. 22, 1387.

Woolfson; M. M. (1961). Direct Methods in Crystallography. Oxford: Univ. Press.

ZACHARIASEN, W. H. (1952). Actn Cryst. 5, 68.

Zachariase:, W. H. (1963). Acta Cryst. 16. 1139.

Acta Cryst. (1968). B24, 35

\title{
The Use of Neutron Anomalous Scattering in Crystal Structure Analysis. I. Non-Centrosymmetric Structures
}

\author{
BY A. K. SINGH AND S. RAMASESHAN \\ Material Sciences Division, National Aeronautical Laboratory, Bangalore-17, India
}

(Received 21 November 1966 and in revised form 6 March 1967)

\begin{abstract}
A niethod for solving the phase problem $a b$ initio in crystal structure studies by neutron diffraction has been suggested. This method is based on the anomalous scattering of thermal neutrons by certain nuclei. Using the data collected at two neutron energies, the process of phase determination is carried out in two steps: (i) the location of the position of the anomalous scatterer and (ii) the correlation of the phase of the structure factor with the phase of the anomalous scatterer. The method gives unique solution of the phases. The expressions deduced arc general and can he used for X-ray anomalous scattering also.
\end{abstract}

\section{Introduction}

Direct methods of sign determination* which are based on the positivity of scattering matter are not applicable to neutron diffraction. Nor can the heavy atom method be used. as the scattering lengths of various nuclei do not differ appreciably. For these reasons the use of neutron diffraction in crystallography has been restricted to the location and the refinement of position

Sce a recent paper by Karle (1966). of light atoms (from the point of view of X-ray scatering) in a structure for which the main features are known from X-ray diffraction work. The possibility of solving the phase problem ah initio in neutron diffraction studies stems from the fact that some nuclei $\left(e . g .{ }^{113} \mathrm{Cd}\right.$, ${ }^{149} \mathrm{Sm},{ }^{151} \mathrm{Eu}$ and $\left.{ }^{157} \mathrm{Gd}\right)$ show anomalous scattering in the thermal neutron range (Peterson \& Smith. 1961, 1962). Ramiseshan (1966) pointed out that anomalous dispersion effects in neutron scattering are much more pronounced than in X-ray scattering and hence can be used effectively in solving the structures provided the 
experimental problems associated with the collection of data are solved. Thus writing the scatteringlength in the form $b=b_{0}+b^{\prime}+i b^{\prime \prime}$, the ratios $b^{\prime} b_{0}$ and $b^{\prime \prime} / b_{0}$ can he as large as 6 and 10 respectively for ${ }^{113} \mathrm{Cd}(f f$. the X-ray case wherc mostly $f^{\prime} / f_{0} \simeq f^{\prime \prime}\left(f_{0} \simeq 0.15\right)$.

Fig. 1 shows that by suitably choosing the neutron energy one can get $\left(b_{0}+b^{\prime}\right) / b_{0} \simeq 7$, for ${ }^{113} \mathrm{Cd}$, i.e. a scattering length which is greater than that of other nuclei by a factor of about 7 . This amounts to having a heavy atom in the structure.

What is perhaps of importance is the fact pointed out by Ramaseshan (1966) that large anomalous dispersion in nuclei like ${ }^{113} \mathrm{Cd},{ }^{149} \mathrm{Sm},{ }^{151} \mathrm{Eu}$ and ${ }^{157} \mathrm{Gd}$ can make it possible to use neutron difraction for solving the structure of large molecules. The success of the anomalous dispersion methods depends on observing significant differences, $\Delta I$, hetueen the intensities of inverse reflexions $h k l$ and $\bar{h} \hat{k} l$. In the case of X-ray anomalous scattering, the structure of vitamin $B_{12}$ mono-acid has been solved with $\langle\Delta I\rangle /\langle I\rangle \simeq 0 \cdot 06$. (人) indicates the root mean square value). If $\langle\Delta I\rangle /\langle I\rangle \simeq$ 0.10 is taken as the criterion for a structure that can be handled by anomalous dispersion methods, it turns out that a structure containing about 2000 atoms per ${ }^{113} \mathrm{Cd}$ can be solved.

The aim of the present paper is to show that by using the data collected at two neutron energies it is possible to locate the position of the anomalous scatterer and determine the phase unambiguously. The results obtained are general and can be applied to X-ray anomalous scattering also.

\section{Determination of the position of the anomalous scatterer}

In handling the phase prohlem by anomalous dispersion methods, the first step is to locate and refine the position of the anomalous scatterer ( $A$-scatterer). The position of A-scatterers which are invariably 'heavy atoms' in the case of X-ray scattering may be determined by Patterson synthesis, but the location of the heavy atom vector in a Patterson synthesis becomes increasingly difficult as the number of light atoms increases. However, in the case of neutron scattering the A-scatterer need not necessarily bc a 'heavy atom' for certain neutron energies and thus location of the $A$ scatterer becomes difficult even in structures of moderate complexity. A method which employs the combination of two sets of data collected at two neutronenergies has been suggested here for locating the position of A-scatterers. The two sets of data arc combined to give $\left|F_{A}\right|^{2}$, the contribution due to the A-scatterer alone. Obviously, a Patterson synthesis with $\left|F_{A}\right|^{2}$ will contain only the A-scatterer vectors. In effect the method is similar to those described by Harding (1962), Kartha \& Parthasarathy (1965), Matthews (1966) and Singh \& Ramaseshan (1966) which employ the combination of isomorphous and anomalous dispersion data. However, the 'two wavelength method' is super- ior to the combination of isomorphous and anoma. lous data because one does not have to depend on the availability of isomorphous pairs. Moreover, lack of exact isomorphism is always a factor to be borne in mind in choosing the isomorphous pairs.

Let us consider a structure with $n_{A}$ A-scatterers, all of the same type and $n_{N}$ normal scatterers ( $N$-scatterer) in the unit cell. Let the scattering length of an A-scat. terer be denoted by

$$
\begin{aligned}
b_{A} & =b_{0}+b^{\prime}+i h^{\prime \prime} \\
& =b(r)+i b(i)
\end{aligned}
$$

where $b(r)=\left(b_{0}+b^{\prime}\right)$ and $b(i)=b^{\prime \prime}$.

The structure factor $\mathbf{F}_{1}(\mathbf{H})$ for neutron-energy $E_{1}$ (as. sociated de Broglie wavelength $\lambda_{1}$ is given by $\lambda_{1}=$ $h / l / 2 E_{1} M, h$ is Planck's constant and $M$ is the mass of the neutron),

where

$$
\mathbf{F}_{1}(\mathbf{H})=\mathbf{F}_{N}+\mathbf{F}_{A 1}+\mathbf{F}_{A 1}^{\prime \prime}
$$

$$
\begin{aligned}
\mathbf{F}_{X} & =\sum_{j=1}^{n_{N}} b_{N j} T_{N j} \exp 2 \pi i \mathrm{H} \cdot \mathbf{r}_{N j} \\
\mathbf{F}_{A 1} & =\sum_{j=1}^{n_{A}} b_{j}(r) T_{A j} \exp 2 \mathrm{niH} \cdot \mathbf{r}_{A j} \\
& =b(r) \sum_{j=1}^{n_{A}} T_{A j} \exp 2 \pi i \mathrm{H}, \mathbf{r}_{A j}
\end{aligned}
$$

since A-scattercrs are all of the same type.

And

$$
\mathbf{F}_{A 1}^{\prime \prime}=b_{1}(i) \sum_{j=1}^{n_{A} A} T_{A j} \exp 2 n i H \cdot \mathbf{r}_{A j}
$$

\section{$\lambda(A)$}

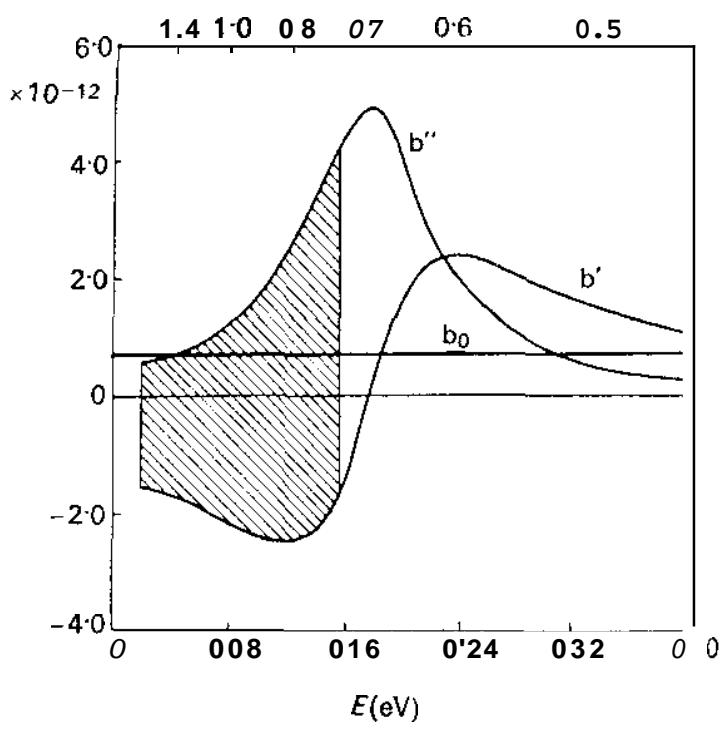

Fig. 1. The variation of $b^{\prime}$ and $b^{\prime \prime}$ for ${ }^{113} \mathrm{C}$ f with wavelength! the vicinity of the resonant wavelength. The shaded pal is shows the region where $E_{1}$ and $E_{2}$ can be chosen convenient" so that $b_{1}(r)=b_{2}(r)$. (See Appendix.) 


$$
\begin{gathered}
T_{N j}=\exp \left(\begin{array}{l}
-B_{N j} \\
\sin ^{2} \theta_{1} \\
\lambda_{1}^{2}-
\end{array}\right) \\
T_{A j}=\exp \left(\begin{array}{c}
\sin ^{2} \theta_{1} \\
\lambda_{1}^{2}
\end{array}\right) .
\end{gathered}
$$

Now

$$
\begin{aligned}
\left|F_{1}(H)\right|^{2}= & \mathbf{F}_{1}(\mathbf{H}) \cdot \mathbf{F}_{1}^{*}(\mathbf{H}) \\
= & \left(\mathbf{F}_{N}+\mathbf{F}_{A 1}+\mathbf{F}_{A 1}^{\prime \prime}\right) \cdot\left(\mathbf{F}_{N}^{*}+\mathbf{F}_{A 1}^{*}+\mathbf{F}_{A 1}^{\prime \prime}\right) \\
= & \left|F_{N}\right|^{2}+\left|F_{A 1}\right|^{2}+\left|F_{A 1}^{\prime \prime}\right|^{2}+2\left|F_{N}\right|\left|F_{A 1}\right| \cos \varphi \\
& \quad+2\left|F_{N}\right|\left|F_{A 1}^{\prime \prime}\right| \cos \varepsilon+2\left|F_{A 1}\right|\left|F_{A 1}^{\prime \prime}\right| \cos \psi
\end{aligned}
$$

where $\varphi, \varepsilon$ and $\psi$ are $\mathbf{F}_{N} \mathbf{F}_{A \perp}$ (angle between $\mathbf{F}_{N}$ and $\left.\mathbf{F}_{A 1}\right) \mathbf{F}_{N} \mathbf{F}_{A 1}^{\prime \prime}$ and $\mathbf{F}_{A 1} \mathbf{F}_{A 1}^{\prime \prime}$ respectively. For only one type of $A$-atom, $\psi=90^{\circ}$ and $\varepsilon=(90-\varphi)$. Thus

$$
\begin{aligned}
& \left|F_{1}(H)\right|^{2}=\left|F_{N}\right|^{2}+\left\{b_{1}^{2}(r)+b_{1}^{2}(i)\right\}|x|^{2} \\
& \quad+2\left|F_{N}\right| b_{1}(r)|x| \cos \varphi+2\left|F_{N}\right| b_{1}(i)|x| \sin \varphi .
\end{aligned}
$$

where

$$
\begin{aligned}
|x|=\left[\left\{\sum_{j=1}^{n_{A}} T_{A j} \cos 2 \pi \mathbf{H}, \mathbf{r}_{A j}\right\}^{2}\right. & \\
& \left.+\left\{\sum_{j=1}^{n_{A}} T_{A j} \sin 2 \pi \mathbf{H} \cdot \mathbf{r}_{A j}\right\}^{2}\right]^{\frac{1}{2}}
\end{aligned}
$$

similarly,

$$
\begin{aligned}
& \left|F_{1}(\bar{H})\right|^{2}=\left|F_{N}\right|^{2}+\left\{b_{1}^{2}(r)+b_{1}^{2}(i)\right\}|x|^{2} \\
& \quad+2\left|F_{N}\right| b_{1}(r)|x| \cos \varphi-2\left|F_{N}\right| b_{1}(i)|x| \sin \varphi .
\end{aligned}
$$

Similar expressions for $\left|F_{2}(H)\right|^{2}$ and $\left|F_{2}(\bar{H})\right|^{2}$ may be written and numbered equations (3) and (4) respectively.

Now we define $\left|F_{m 1}(H)\right|^{2},\left|F_{m 2}(H)\right|^{2}, \Delta I_{1}$, and $\Delta I_{2}$ as follows,

$$
\begin{aligned}
\left|F_{m_{1}}(H)\right|^{2}= & \frac{1}{2}\left[\left|F_{1}(H)\right|^{2}+\left|F_{1}(\bar{H})\right|^{2}\right] \\
= & \left|F_{N}\right|^{2}+\left\{b_{1}^{2}(r)+b_{1}^{2}(i)\right\}|x|^{2} \\
& +2\left|F_{N}\right| b_{1}(r)|x| \cos \varphi
\end{aligned}
$$

$$
\begin{aligned}
\mid F_{\left.m_{2}(H)\right|^{2}}= & \frac{1}{2}\left[\left|F_{2}(H)\right|^{2}+\left|F_{2}(\bar{H})\right|^{2}\right] \\
= & \left|F_{N}\right|^{2}+\left\{b_{2}^{2}(r)+b_{2}^{2}(i)\right\}|x|^{2} \\
& +2\left|F_{N}\right| b_{2}(r)|x| \cos \varphi \\
\Delta I_{1}= & {\left[\left|F_{1}(H)\right|^{2}-\left|F_{1}(\bar{H})\right|^{2}=\right.} \\
& 4\left|F_{N}\right| b_{1}(i)|x| \sin \varphi
\end{aligned}
$$

and

$$
\begin{aligned}
& \Delta I_{2}=\left[\left|F_{2}(H)\right|^{2}-\left|F_{2}(\bar{H})\right|^{2}\right]= \\
& 4\left|F_{N}\right| b_{2}(i)|x| \sin \varphi .
\end{aligned}
$$

Combining equations (7) and (8) we get,

$$
\frac{\Delta I_{1}}{2 b_{1}(i)}=-\frac{\Delta I_{2}}{2 b_{2}(i)}=2\left|F_{N}\right||x| \sin \varphi=\delta .
$$

It may be noted that $\Delta I_{1} / \Delta I_{2}=b_{1}(i) / b_{2}(i)=$ constant. Since the absorption is quite different for the two wavelengths, this will provide a check on the accuracy of the data.

The first term in equation (5) gives rise to $N-N$ peaks in a Patterson synthesis computed with $\left|F_{m 1}(H)\right|^{2}$ as coefficient; the second and third terms give rise to $A-A$ and $A-N$ peaks respectively. In order to be able to locate the $\boldsymbol{A}-\boldsymbol{A}$ peaks it is essential to eliminate $\boldsymbol{A}-\boldsymbol{N}$ and $\mathbf{N}-\mathbf{N}$ peaks which tend to mask $\boldsymbol{A}-\boldsymbol{A}$ peaks. Ramaseshan (1966) suggested that if the neutron energies are so chosen that $b_{1}(r)=-b_{2}(r)$, then a Patterson function with $\left[\left|F_{m_{1}}(H)\right|^{2}+\left|F_{m_{2}}(H)\right|^{2}\right]$ as coefficient will contain only $\boldsymbol{A}-\boldsymbol{A}$ and $N-N$ peaks; the background due to $A-N$ peaks will be eliminated. However, a simple estimation shows that in a structure containing a large number of $N$-atoms, $N-N$ peaks give rise to a background which is more serious than that due to $A-N$ peaks. It is therefore necessary to eliminate both $A-N$ and $\mathbf{N}-\mathbf{N}$ peaks.

\section{Expressionfur $|x|^{2}$}

We shall now derive an expression for $|x|^{2}$ in terms of $\left|F_{m_{1}}(H)\right|^{2},\left|F_{m_{2}}(H)\right|^{2}, \delta, b_{1}(r), b_{1}(i), b_{2}(r)$ and $b_{2}(i)$.

Eliminating $\varphi$ between equations (5) and (7) and using equation (9) we get,

$$
\begin{aligned}
& \left|F_{N}\right|^{4}-2\left|F_{N}\right|^{2}\left[\left|F_{m_{1}}(H)\right|^{2}+\left\{b_{1}^{2}(r)-b_{1}^{2}(i)\right\}|x|^{2}\right] \\
& +\left[\left|F_{m_{1}}(H)\right|^{2}-\left\{b_{1}^{2}(r)+b_{1}^{2}(i)\right\}|x|^{2}\right]^{2}+\delta^{2} b_{1}^{2}(r)=0 .
\end{aligned}
$$

Similarly equations (6), (8) and (9) give,

$$
\begin{aligned}
& \left|F_{N}\right|^{4}-2\left|F_{N}\right|^{2}\left[\left|F_{m_{2}}(H)\right|^{2}+\left\{b_{2}^{2}(r)-b_{2}^{2}(i)\right\}|x|^{2}\right] \\
& +\left[\left|F_{m_{2}}(H)\right|^{2}-\left\{b_{2}^{2}(r)+b_{2}^{2}(i)\right\}|x|^{2}\right]^{2}+\delta^{2} b_{2}^{2}(r)=0 .
\end{aligned}
$$

Subtracting equation (11) from (10) gives,

$$
\begin{aligned}
& 2\left|F_{N}\right|^{2}\left[\left\{\left|F_{m_{1}}(H)\right|^{2}-\left|F_{m_{2}}(H)\right|^{2}\right\}+\left\{\left(b_{1}^{2}(r)-b_{1}^{2}(i)\right)\right.\right. \\
& \left.\left.-\left(b_{2}^{2}(r)-b_{2}^{2}(i)\right)\right\}|x|^{2}\right]=\left[\left|F_{m_{1}}(H)\right|^{2}-\left\{b_{1}^{2}(r)+b_{1}^{2}(i)\right\}|x|^{2}\right]^{2} \\
& -\left[\left|F_{m_{2}}(H)\right|^{2}-\left\{b_{2}^{2}(r)+b_{2}^{2}(i)\right\}|x|^{2}\right]^{2}+\delta^{2}\left\{b_{1}^{2}(r)-b_{2}^{2}(r)\right\} .
\end{aligned}
$$

Now multiplying equations (5) and (6) by $b_{2}(r)$ and $b_{1}(r)$ respectively and subtracting the resulting equations we have,

$$
\begin{aligned}
& {\left[b_{2}(r)\left|F_{m 1}(H)\right|^{2}-b_{1}(r)\left|F_{m 2}(H)\right|^{2}\right]=} \\
& \left\{b_{2}(r)-b_{1}(r)\right\}\left|F_{N}\right|^{2}+\left[b_{2}(r)\left\{b_{1}^{2}(r)+b_{1}^{2}(i)\right\}\right. \\
& \left.-b_{1}(r)\left\{b_{2}^{2}(r)+b_{2}^{2}(i)\right\}\right]|x|^{2} .
\end{aligned}
$$

Eliminating $\left|F_{N}\right|^{2}$ between (12) and (13) we have,

where

$$
P|x|^{4}-2 Q|x|^{2}+R=0 \text {, }
$$

$$
\begin{aligned}
& \mathrm{P}=\left\{b_{1}(r)-b_{2}(r)\right\}^{2}\left[2\left\{b_{1}^{2}(i)+b_{2}^{2}(i)\right\}\right. \\
& \left.+\left\{b_{1}(r)-b_{2}(r)\right\}^{2}\right]+\left\{b_{1}^{2}(i)-b_{2}^{2}(i)\right\}^{2} \\
& \mathrm{Q}=\left\{b_{1}(r)-b_{2}(r)\right\}^{2}\left[\left|F_{m_{1}}(H)\right|^{2}+\left|F_{m_{2}}(H)\right|^{2}\right] \\
& +\left\{b_{1}^{2}(i)-b_{2}^{2}(i)\right\}\left\{\left.F_{m_{1}}(H)\right|^{2}-\left|F_{m_{2}}(H)\right|^{2}\right\} \\
& R=\left\{\left|F_{m_{1}}(H)\right|^{2}-\left|F_{m_{2}}(H)\right|^{2}\right\}^{2}+\delta^{2}\left\{b_{1}(r)-b_{2}(r)\right\}^{2} \text {. }
\end{aligned}
$$

It is obvious that $\mathrm{P}, \mathrm{Q}$ and $R$ are always positive and therefore equation (14) will always have two positive roots $\left|x_{+}\right|^{2}$ and $\left|x_{-}\right|^{2}$ given by

$$
\left|x_{ \pm}\right|^{2}=Q / P \pm\left(Q^{2}-R P\right)^{\frac{1}{2}} / P \text {. }
$$

If we substitute $b_{2}(r)=b_{2}(i)=0$, it is equivalent to combining isomorphous and anomalous dispersion data. In this case equation (15) reduces to equation (11) of Singh \& Ramaseshan (1966). 


\section{Interpretation of two solutions}

The physical significance of the tuo values of $|x|^{2}$ as obtained from equation (14) is that, in general, there are two sets of values of $\left.|x| . \mid F_{N}\right\}$ and $\varphi$ for a given set of $\left|F_{1}(H)\right|^{2},\left|F_{1}(\bar{H})\right|^{2},\left|F_{2}(H)\right|^{2}$ and $\left|F_{2}(\bar{H})\right|^{2}$. Two possible values of $\left|F_{N}\right|$ and $\varphi$ can be calculated as follows:

Subtracting equation (6) from (5) we get.

$$
\begin{aligned}
& \left|F_{m_{1}}(H)\right|^{2}-\left|F_{m_{2}}(H)\right|^{2}= \\
& {\left[\left\{b_{1}^{2}(r)+b_{1}^{2}(i)\right\}-\left\{b_{2}^{2}(r)+b_{2}^{2}(i)\right\}\right]|x|^{2}} \\
& +2\left|F_{N} \| x\right|\left\{b_{1}(r)-b_{2}(r)\right\} \cos \varphi \\
& \left|F_{N}( \pm)\right| \cos \varphi( \pm)= \\
& {\left[\left\{\left|F_{m 1}(H)\right|^{2}-\mid F_{m_{2}}(H)\right\}^{2}\right\}-\left\{\left(b_{1}^{2}(r)+b_{1}^{2}(i)\right)\right.} \\
& \left.\left.-\left(b_{2}^{2}(r)+b_{2}^{2}(i)\right)\right\}\left|x_{ \pm}\right|^{2}\right] / 2\left[b_{1}(r)-b_{2}(r)\right]\left|x_{ \pm}\right|,
\end{aligned}
$$

and from equation (9),

$$
\left|F_{N}( \pm)\right| \sin \varphi( \pm)=\delta / 2\left|x_{ \pm}\right|
$$

Thus the values of $\left|F_{N}\right|$ and $\varphi$ corresponding to $\left|x_{+}\right|$ and $\left|x_{-}\right|$can he obtained from equations (16) and (17).

For illustration computations were made in a hypothetical case with $b_{1}(r)=2 \cdot 0, b_{1}(i)=1 \cdot 0, b_{2}(r)=1 \cdot 0$, $b_{2}(i)=1 \cdot 0, \varphi=60^{\circ},\left|F_{N}\right|=4.0$ and $|x|=0.50$. Thesegive $\left\{F_{1}(H)\right\}=4.972,\left\{F_{1}(\bar{H})\right\}=4 \cdot 217,\left\{F_{2}(H) \mid=4 \cdot 686,\left\{F_{2}(\bar{H})\right\}\right.$ $=3.878$. Thus, if we start with these as the values of the observed structure amplitudes we get from equations (15), (16) and (17) $\left|x_{+}\right|=3.95,\left|F_{N}(+)\right|=5 \cdot 581$, $\varphi(+)=175^{\circ} 18^{\prime}$ and $\left|x_{-}\right|=0.50,\left|F_{N}(-)\right|=4.0, \varphi(-)=$ $60 "$.

\section{Choice of the correct solution}

The next step is to choose the correct solution of equation (14). If the structure contains $n t$ anomalous scatterers (all of the same type), then $\left|x_{m}\right|^{2}$, the maximum possible value of $|x|^{2}$ is $n_{A}^{2}$ uhen $n_{f}$ nuclei scatter all in phase. Thus if $\left|x_{+}\right|^{2}>n_{A}^{2}$ the only acceptable solution is $\left|x_{-}\right|^{2}$. If, however, $\left|x_{+}\right|^{2}<n_{A}^{2}$, both $\left|x_{+}\right|^{2}$ and $\left|x_{-}\right|^{2}$ are acceptable solutions by this criterion. In such cases the ambiguity remains unresolved.

As the quantity $\left(Q^{2}-P R\right)$ tends to zero, $\left|x_{+}\right|^{2}$ and $|x|^{2}$ tend to be equal. One may come across cases where $\left|x_{+}\right|^{2}$ rind $\left|x_{-}\right|^{2}$ are nearly equal. This makes the selection of the correct root difficult. In such cases it is better to take $Q / P$, the mean value of the two roots for $|x|^{2}$.

\section{Unique solution of $|x|^{2}$}

If the two neutron-energies arc so chosen that $b_{1}(r)=$ $b_{2}(r)$ and $b_{1}(i) \neq b_{2}(i)$ (it is clear from Fig. 1 that such a choice is certainly possible) then

$$
\text { and } R=\left\{\left|F_{m_{1}}(H)\right|^{2}-\left|F_{m_{2}}(H)\right|^{2}\right\}^{2}
$$

$$
\begin{aligned}
& P=\left\{b_{1}^{2}(i)-b_{2}^{2}(i)\right\}^{2} \\
& Q=\left\{b_{1}^{2}(i)-b_{2}^{2}(i)\right\}\left\{\left|F_{m_{1}}(H)\right|^{2}-\left|F_{m_{2}}(H)\right|^{2}\right\}
\end{aligned}
$$

This leads to $\left(Q^{2}-R P\right)=0$ and therefore the two roots are coincident and are given by,

$$
\left|x_{+}\right|^{2}=\left|x_{-}\right|^{2}=Q / P \text {. }
$$

It may be noted that this result* can be obtainer directly by subtracting equation (6) from equation ( 5 )

Owing to the practical difficulty in selecting the neutron-energies $E_{1}$ and $E_{2}$ for which $b_{1}(r)=b_{2}(r)$, one may have a case $b_{1}(r) \simeq b_{2}(r)$. It is rather fortunate tha: the factor $\left\{b_{1}(r)-b_{2}(r)\right\}$ occurs in the expressions 0 $P, Q$ and $R$ as squares and, therefore, equation (1) can be used without introducing much error even ${ }_{11}$ $b_{1}(r)$ and $b_{2}(r)$ are slightly different.

As $\mathrm{P}$ depends only on the scattering lengths it $\mathrm{ma}$ ! appear that $P$ can be made zero by suitably selectin: $E_{1}$ and $E_{2}$. In such a case equation (14) will have onl: one root $|x|^{2}=R / 2 Q$. However, since $P$ is the sum 0 three positive terms. it can be made zero only wher the three terms are separately zero which cannot be done except in a trivial case $E_{1}=E_{2}$.

\section{Refmement of the thermal and positional parameters}

Once $|x|$ is determined, a Patterson function witt: $|x|^{2} b_{1}^{2}(r)$ as coefficients will give the positions of A-scat. terers. A comparison of $|x|$ calculated from the know: positions of A-scatterers uith those obtained frot equation (15) will reveal the reflexions for which thi root has been wrongly chosen. If equation (17) is usei this comparison is not necessary. The values of $|x| 0 \mathrm{~b}$ tained this uay can be used to refine the thermal an: positional parameters of A-scatterers.

\section{Unique soiution of the phases}

It can be easily shown (Ramachandran \& Raman 1956) that the phase $\alpha_{A_{1}}(H)$ of the A-scatterer contr bution to the structure factor is related to the phast $\alpha_{1}^{\prime}(H)$ of $\left|F_{1}^{\prime}(H)\right|$ by,

$$
\alpha_{1}^{\prime}(H)=\alpha_{A_{1}}+\theta_{1}
$$

(see Fig.2) where $\theta_{1}$ is given by

$$
\sin 8,=\Delta I_{1} / 4\left|F_{1}^{\prime}(H)\right|\left|F_{A 1}^{\prime \prime}\right|=\delta / 2\left|F_{1}^{\prime}(H) \| x\right|
$$

and

$$
\left|F_{1}^{\prime}(H)\right|=\left[\frac{1}{2}\left\{\left|F_{1}(H)\right|^{2}+\left|F_{1}(\bar{H})\right|^{2}\right\}-\left|F_{A 1}^{\prime \prime}\right|^{2}\right]^{\prime}
$$

$\theta_{1}$ determined from equation (19) will have tim values $\theta_{1}$ and $(180 "-8$,). Thus there is a twofold an biguity in $\alpha_{1}^{\prime}(H)$ calculated from equation (18). In X-rd anomalous dispersion work this ambiguity has bet resolved by various indirect methods (Ramaseshar 1963).

Here it has been shown that the use of data collect: attwoneutron energiesyields a urique solution of $\alpha_{1}^{\prime}(H)$ Referring to Fig. 2. $\varphi$ and $\theta_{1}$ are related by,

or

$$
\begin{aligned}
& \left|F_{A_{1}}\right| / \sin \left(\varphi-\theta_{1}\right\}=\left|F_{1}^{\prime}(H)\right| / \sin \varphi \\
& \sin \left(\varphi-\theta_{1}\right)=\left|F_{A_{1}}\right| /\left|F_{1}^{\prime}(H)\right| \backslash \operatorname{in} \varphi
\end{aligned}
$$

* The authors are grateful to the referec for drawing the: attention to the fact that when $b_{1}(r)=b_{2}(r)$, a difference eve Patterson function (Okaya, Saito \& Pepinsky, 1955) also lear to similar results. 


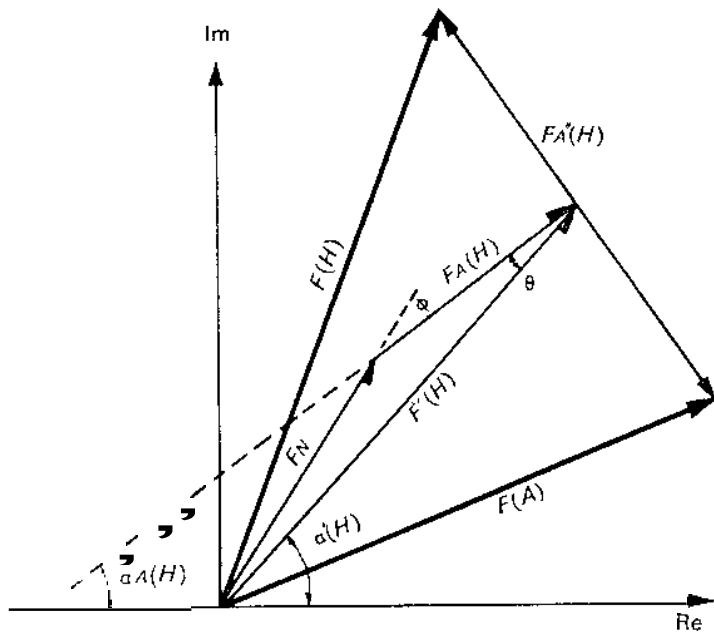

Fig. 2. Argand diagram representing $\mathbf{F}(\mathbf{H})$ and $\mathbf{F}(\overline{\mathbf{H}})$.

Combining equations (19) and (20) we get $\cos \theta_{1}=\left[\delta \cot \varphi / 2|x|+\left|F_{A_{1}}\right|\right] /\left|F_{1}^{\prime}(H)\right|$.

Equations (5), (6) and (9) can be combined to give $\cot \varphi=\left[\left\{\left|F_{m 1}(H)\right|^{2}-\left|F_{m 2}(H)\right|^{2}\right\}-\left\{\left(b_{1}^{2}(r)+b_{1}^{2}(i)\right)\right.\right.$ $\left.\left.-\left(b_{2}^{2}(r)+b_{2}^{2}(i)\right)\right\}|x|^{2}\right] /\left\{b_{1}(r)-b_{2}(r)\right\} \dot{\delta}$.

On substituting the value of $\cot \varphi$ from equation (22) in equation (21) we have,

$$
\cos \mathbf{0}_{r}=\begin{gathered}
\left\{\left|F_{m_{1}}(H)\right|^{2}-\left|F_{m_{2}}(H)\right|^{2}\right\}-\left\{\left(b_{1}^{2}(r)+b_{1}^{2}(i)\right)-\left(b_{2}^{2}(r)+b_{2}^{2}(i)\right)\right\}|x|^{2} \\
2\left\{b_{1}(r)-b_{2}(r)\right\}\left|F_{1}^{\prime}(H)\right||x|
\end{gathered}+\frac{\left|F_{A_{1}}\right|}{\left|F_{1}^{\prime}(H)\right|^{\prime}} .
$$

In case $b_{1}(r)=b_{2}(r)$, equations (5) and (6) give,

$$
\left|F_{m_{1}}(H)\right|^{2}-\left.F_{m_{2}}(H)\right|^{2}=\left\{b_{1}^{2}(i)-b_{2}^{2}(i)\right\}|x|^{2}
$$

and equation (23) reduces to,

$$
\cos \theta_{1}=\frac{-\left\{b_{1}(r)+b_{2}(r)\right\}|x|+2\left|F_{A 1}\right|}{2\left|F_{1}^{;}(H)\right|}
$$

Since $\sin \theta_{1}$ and $\cos \theta_{1}$ are known from equations (19) and (23) respectively, $\theta_{1}$ and hence $\alpha_{1}^{\prime}(H)$ is known.

Similarly $\alpha_{2}(H)$ can also be obtained.

\section{Conclusion}

Thus we see that combination of the data collected at two neutron-energies makes it possible to determine and refine the thermal and positional parameter of the anomalous scatterer in large molecules - a process which is normally difficult. Further, the phases of the reflexions can be determined unambiguously.

The author's thanks are due to the referee for his belpful comments.

\section{APPEXDIX}

It has been shown in $\$ 2$ that if $E_{1}$ and $E_{2}$ are so chosen that $b_{1}(r)=b_{2}(r)=b(r)$ and $b_{1}(i) \neq b_{2}(i),|x|^{2}$ can be determined uniquely. A method has been indicated to calculate such pairs of $E_{1}$ and $E_{2}$.

Measurement of the scattering and absorption crosssection of $11 \mathrm{Cd}$ and ${ }^{149} \mathrm{Sm}$ (Brockhouse, 1953) shows that the one-level Breit-Wigner formula is valid. It can be shown from the one-level Breit-Wigner theory that,

and

$$
b^{\prime}=\frac{1}{2} \begin{aligned}
& g w \hat{\lambda}_{0} \Gamma_{n}\left(E-E_{0}\right) \\
& \left(E-\overline{E_{0}}\right)^{2}+\Gamma^{2 / 4}
\end{aligned}
$$

$$
b^{\prime \prime}=b(i)=\frac{1}{4} \quad \begin{gathered}
g_{11} \lambda_{0} \Gamma_{n} \Gamma \\
\left(E-E_{0}\right)^{2}+\Gamma^{2} / 4
\end{gathered} .
$$

where $g$ is a spin weighting factor, $w$ is the isotopic abundance, which is unity for a resonant isotope, $\lambda_{0}$ is the wavelength at resonance divided by $2 \pi, \Gamma_{n}$ is the neutron width, $\Gamma$ is the total width, $\mathrm{C}$ is the energy of measurement and $E_{0}$ the resonance energy. Substituting the numerical values (Brockhouse, 1953) for various parameters in equations (i) and (ii) we get

and

$$
b^{\prime}=\frac{A\left(E-E_{0}\right)}{\left(E-E_{0}\right)^{2}+B}
$$

$$
b^{\prime \prime}=\frac{\mathrm{C}}{\left(E-E_{0}\right)^{2}}+B,
$$

where $A, B, C$ and $E_{0}$, have values respectively $0.278 \times$ $10^{-12}, 0.0032$. $1.565 \times 10^{-14}$ and $0.178 \mathrm{eV}$ for ${ }^{113} \mathrm{Cd}$ and $0.250 \times 10^{-12}, 0.0014,0.920 \times 10^{-14}$ and $0.096 \mathrm{eV}$ for ${ }^{149} \mathrm{Sm}$.

The two sets of values of $E$ for which $b_{1}(r)=b_{2}(r)=b^{\prime}$ can be calculated from the equations and

$$
\left(E_{1,2}-E_{0}\right)=
$$

$$
0 \cdot 139 / b^{\prime} \pm\left[\left\{0 \cdot 139 / b^{\prime}\right\}^{2}-0.0034\right]^{\frac{3}{2}} \text { for }{ }^{111} \mathrm{Cd}
$$

$$
\left(E_{1,2}-E_{0}\right)=
$$

$$
0 \cdot 125 / b^{\prime} \pm\left[\left\{0 \cdot 125 / b^{\prime}\right\}^{2}-0 \cdot 0014\right]^{1} \text { for }{ }^{149} \mathrm{Sm} \text {. }
$$

It is clear from Fig.1 that such a pair, $E_{1}$ and $E_{2}$, can he chosen on either side of the resonance energy $\left(E_{0}=0.178\right)$. However, the smaller-energy side (the shaded region) is preferable to the greater-energy side because of the convenient working wavelenpth and the large flux of neutrons from the pile. This reyion corresponds to the X-ray wavelength range from Mo $K \alpha$ to $\mathrm{Fc} K \alpha$.

\section{References}

Brockhouse, B. N. (1953). Canad. J. Physics, 31, 432.

Harding, M. M. (1962). Ph.D. Thesis, Oxford University. Karle, J. (1966). Acta Ciyst. 20, 881.

Kartha,G.\& Parthasarathy,R. (1965). Acta Cryst. 18, 745. Matthews, B.W. (1966). Acta Cryst. 20, 230.

Okaya, Y. Satto, Y. \& Pepinsky, R, (1965). Phys. Rev. 98, 1857.

Peterson, S. W. \& Smith, H. G. (1961). Phys. Rev. Letters, $6,7$.

Peterson, S. W. \& Smith, H. G. (1962). J. Phys. Soc. Japan, 17, 335 .

RamaChandran, G.N.\& RaMan, S. (1956).Cur.Sci. 25, 348.

Ramaseshav, S. (1963). Advanced Methods in X-ray Crystallography. London: Academic Press.

Ramaseshan, S. (1966). Curr. Sci. 35, 87.

Singh, A. K. \& Rainaseshan, S. (1966). Acta Cryst. 21, 279. 